\title{
Low-energy Cosmic Rays in Supernova Remnants Interacting with Molecular Clouds
}

\author{
Kumiko K. Nobukawa* \\ Department of Physics, Faculty of Science, Nara Women's University, \\ Kitauoyanishi-machi,Nara, Nara 630-8506, Japan \\ E-mail: kumikodcc.nara-wu.ac.jp
}

\begin{abstract}
Information on low-energy cosmic rays (CRs) below the $\mathrm{MeV}$ band has been very limited due to difficulty of measurements in contrast to high-energy $(\mathrm{GeV}-\mathrm{TeV}) \mathrm{CRs}$, which are observable via gamma-rays. Only ionization rate provides indirect information on low-energy CRs. The neutral iron line at $6.4 \mathrm{keV}$ can be a new effective probe. Utilizing the Suzaku archive data, we searched supernova remnants (SNRs) for the particle-induced $6.4 \mathrm{keV}$ line. Since accelerated CR particles can produce the $6.4 \mathrm{keV}$ line when they interact with molecular clouds, we focused on SNRs interacting with molecular clouds. The $6.4 \mathrm{keV}$ line probably induced by CR particles has been found from more than 10 SNRs. More than a half of the SNRs also show gamma-ray emission. We found that both the $6.4 \mathrm{keV}$ line and gamma-ray emissions can be explained by a CR escaping scenario at least for W28, W44, and IC 443. It indicates that low-energy CRs producing the $6.4 \mathrm{keV}$ line have been accelerated at the SNRs together with high-energy CRs.
\end{abstract}

Multifrequency Behaviour of High Energy Cosmic Sources - XIII - MULTIF2019

3-8 June 2019

Palermo, Italy

\footnotetext{
* Speaker.
} 


\section{Introduction}

Supernova remnants (SNRs) are considered that they accelerate cosmic rays (CRs) via shock acceleration. When high-energy CRs interact with molecular clouds (MCs), they emit gammarays, so they can be probed by gamma-rays [ [U, []]. On the other hand, low-energy CRs have been a missing link of acceleration mechanism because they cannot be probed by the gamma-ray observations in principle. Only ionization rate provides indirect information on low-energy CRs [B], Direct observations of low-energy CRs are significantly affected by solar modulation. Robust direct measurements are performed by the Voyager mission, in which the spectra of low-energy CRs have been observed outside the solar system for the first time [䧃].

We consider that the neutral iron line can be an effective prove. When low-energy CR protons or electrons interact with cold gas, neutral iron line at $6.4 \mathrm{keV}$ is produced. Especially, CRs with the energies of $10 \mathrm{MeV}$ for protons and $10 \mathrm{keV}$ for electrons are most effective to create the iron line because of large cross section. Since iron has high abundance in the interstellar medium $\left([\mathrm{H}] /[\mathrm{Fe}] \sim 3 \times 10^{-5}\right.$; [] $]$ ) and high fluorescence yield $(\sim 0.3)$, the $6.4 \mathrm{keV}$ line is generated with the highest intensity among X-ray fluorescence from other neutral heavy atoms. Furthermore, the $\mathrm{X}$-ray continuum is produced via bremsstrahlung or inverse bremsstrahlung at the same time, and its intensity depends on the projectiles: electrons produce much more continuum than protons. Therefore, the equivalent width of the $6.4 \mathrm{keV}$ line, that is the intensity ratio of the line and continuum, distinguishes the projectile. We have searched SNRs interacting with MCs for the $6.4 \mathrm{keV}$ line induced by low-energy CRs.

\section{Observations}

We utilized the archive data of Suzaku, which were operated in 2005-2015. Suzaku is even now the best detector for diffuse iron $\mathrm{K}$-shell line emissions because it has a large effective area in the iron K-shell band and also a good energy resolution enough to distinguish the neutral iron line from the He-like iron line. And it has an advantage of low and stable background for diffuse emissions. Actually, the probably particle-induced $6.4 \mathrm{keV}$ line was already found in 3C391 and Kes 79 from the Suzaku data $[$ [, 0$]$. They were the only SNRs from which the particle-induced line was found until this study.

\section{Observation results}

We reported in 2018 that the $6.4 \mathrm{keV}$ line is found from five SNRs, W28, W44, Kes 67, Kes 69, and Kes $78[8]$. Figure 1 is a stacked spectrum of these SNRs from which a background spectrum is already subtracted. We can see a clear line emission at $6.4 \mathrm{keV}$. All the SNRs are known to be interacting with MCs [9]. One possibility of the origin of $6.4 \mathrm{keV}$ line is of the thermal plasma. In fact, some young SNRs exhibit an iron line near $6.4 \mathrm{keV}$ radiated from the iron ejecta. However, we interpret that the $6.4 \mathrm{keV}$ line would not be of the plasma origin. The flux of the iron K-shell line due to excitation or ionization by thermal electrons rapidly decrease with the electron temperature of below $1 \mathrm{keV}$. Since the electron temperature of all our samples is below $1 \mathrm{keV}$, it is almost impossible for our samples to emit a detectable iron K-shell line. Alternatively, the $6.4 \mathrm{keV}$ line 
can be fluorescence from MCs ionized by X-rays or low-energy CRs. We concluded that the CRs are the most plausible because no bright X-ray source exists near each SNR. In the CR scenario, the projectile is distinguished by equivalent width of the $6.4 \mathrm{keV}$ line. Proton bombardment results in the equivalent width of $\sim 1 \mathrm{keV}$ whereas electron bombardments does in that of $<0.4 \mathrm{keV}$ [ए]]]. The observed value in the five SNRs is $1.0_{-0.4}^{+0.7} \mathrm{keV}$, which favors the proton origin.

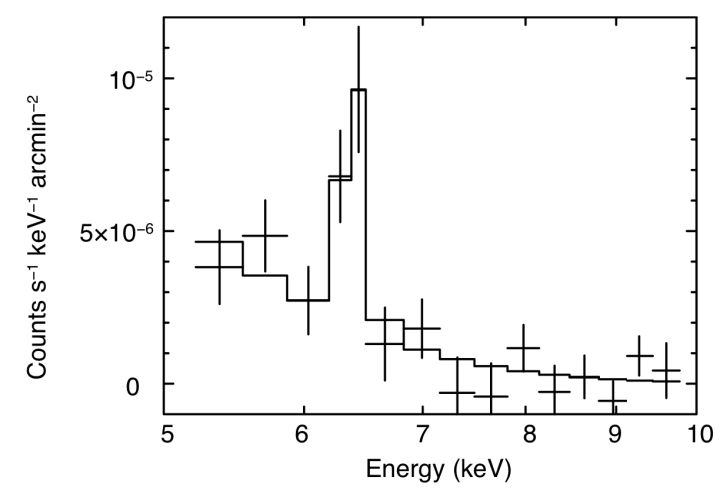

Figure 1: Stacked spectrum of the five SNRs, W28, W44, Kes 67, Kes 69, and Kes 78, from which a background spectrum is already subtracted.

After our paper in 2018, the number of samples is increasing now; more than 10 SNRs (including previously found ones, 3C391 and Kes 79) shows the $6.4 \mathrm{keV}$ line probably induced by CR

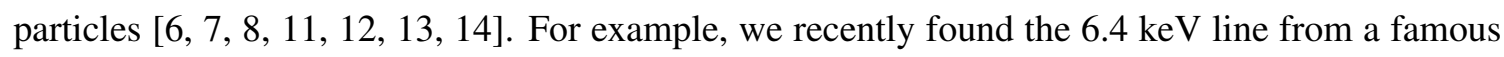
SNR, IC 443, with $3.8 \sigma$ as reported in Nobukawa et al. (2019b) [प4]]. The object's view angle is as large as $\sim 0 .{ }^{\circ} 5$, and thus it is suitable for studying the morphology of the $6.4 \mathrm{keV}$ line emission. Figure 2 indicates the distributions of the $6.4 \mathrm{keV}$ line intensity (blue) and thermal emission (yellow; the 1-3 keV band), which is overlapped by that of ${ }^{12} \mathrm{CO}$ (contours; S. Yoshiike private communication). The spatial distribution of the $6.4 \mathrm{keV}$ line intensity seems to be different from that of the thermal emission. It implies that the $6.4 \mathrm{keV}$ line emission does not originate from the thermal plasma. In fact, the IC 443's plasma is known to be in the recombining state [प5, [6]], and it is impossible that the recombining plasma radiates a detectable $6.4 \mathrm{keV}$ line. The $6.4 \mathrm{keV}$ line intensity is associated with the MCs. There is no bright X-ray source enough to explain the line intensity. These facts indicate that the low-energy CR origin is most plausible. The equivalent width of $6.4 \mathrm{keV}$ was measured to be $>1.2 \mathrm{keV}$, which favors the proton projectiles. There is a spatial correlation between the brightest regions of the $6.4 \mathrm{keV}$ line and the $\mathrm{GeV}-\mathrm{TeV}$ emission as well as the ${ }^{12} \mathrm{CO} \mathrm{MC}$ emission. This 3 -fold correlation favors a hadronic rather than a leptonic origin for the $\mathrm{GeV}-\mathrm{TeV}$ gamma-ray emission.

\section{Comparison with Gamma-rays}

More than a half of the SNRs accompanied with the $6.4 \mathrm{keV}$ line show gamma-ray emission (table 1 of [ㅍ]]). In fact, we found that both the $6.4 \mathrm{keV}$ line and gamma-ray emissions can be explained by a CR escaping scenario. This study was first conducted by Makino et al. (2019) [ए]] 


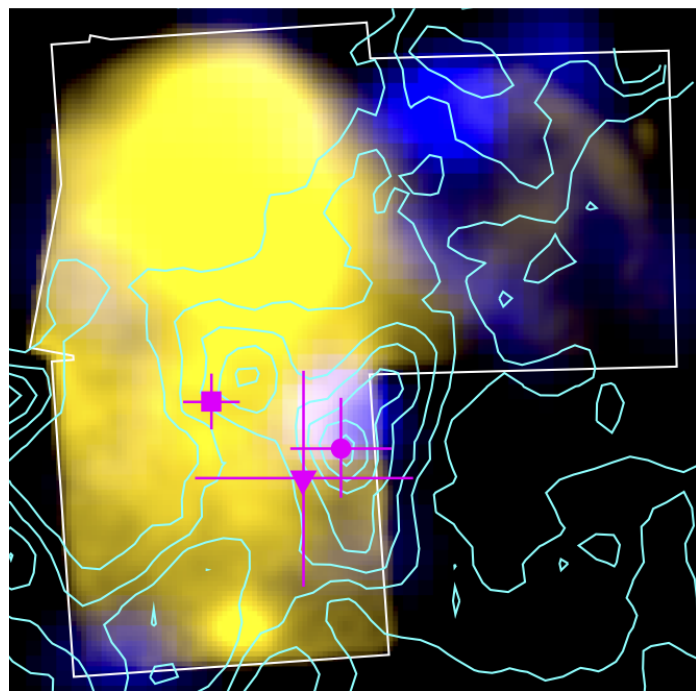

Figure 2: X-ray images of IC 443: distributions of the $6.4 \mathrm{keV}$ line intensity (blue) and thermal emission (yellow; the 1-3 keV band), which are overlapped by that of ${ }^{12} \mathrm{CO}(J=1-0)$ at $-18-+10 \mathrm{~km} \mathrm{~s}^{-1}$ (contours; $\mathrm{S}$. Yoshiike private communication). White lines indicate the fields of views of Suzaku. The magenta marks and crosses represent the centroids of gamma-ray sources observed by Fermi (square), MAGIC (circle), and VERITAS (triangle) and their position errors [ए9, 20, 20]].

Table 1: Comparison of the $6.4 \mathrm{keV}$ line intensity calculated with the CR escaping model [ए8], and the observed ones in W28, W44, and IC 443.

\begin{tabular}{lcc}
\hline Object & \multicolumn{2}{c}{ Intensity of the 6.4 keV line* } \\
& Model & Observations \\
\hline W28 & 0.08 & $0.10 \pm 0.05^{\dagger}$ \\
W44 & 0.13 & $0.15 \pm 0.08^{\dagger}$ \\
IC 443 & 0.29 & $0.27 \pm 0.12^{\S}$ \\
\hline
\end{tabular}

${ }^{*}$ In the unit of photon $\mathrm{s}^{-1} \mathrm{~cm}^{-2} \mathrm{sr}^{-1}$.

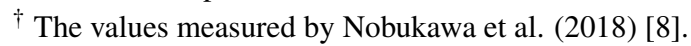

$\S$ The values measured by Nobukawa et al. (2019b) [[प]].

for W28 and W44, and Nobukawa et al. (2019b) [144] applied the model to IC 443. Gamma-ray emission from those three SNRs is considered to be the hadronic origin.

In our model, high-energy CRs start to escape from SNRs first. When the high-energy CRs interact with MCs, they emit gamma-rays. On the other hand, MeV CRs are still confined around the shock and not escape yet. When the shock contacts with the MCs, MeV CRs interact with MCs and emit the $6.4 \mathrm{keV}$ line. The distribution of high-energy CRs is calculated based on a standard scenario of CR escape for SNRs [D2]. And also gamma-ray spectrum from MCs is calculated. This model well reproduces the gamma-ray observations of the three SNRs, W28, W44, and IC 443. Using those spectra, model parameters for CRs are constrained. For the $6.4 \mathrm{keV}$ line, the model supposes that MeV CRs seep into MCs when the shock contacts with the MCs and MeV CRs are 
cooled by ionization. Column density of MeV CRs is represented by the product of shock velocity, number density of CRs and cooling time. The intensity of the $6.4 \mathrm{keV}$ line is in proportion to the column density of $\mathrm{MeV}$ CRs. Using the parameters of CRs obtained by fitting the gamma-ray spectra, we calculated the intensities of the $6.4 \mathrm{keV}$ line. The expected intensities are consistent with our observations for all the three samples as summarized in table 1.

The conclusion that the $6.4 \mathrm{keV}$ line and gamma-ray emissions from the SNRs can be explained by an CR escaping scenario may not be an amazing result, but our study demonstrated that the observations of the $6.4 \mathrm{keV}$ line indeed made it possible to study CRs including the low-energy band.

\section{DISCUSSION}

JAMES HOWARTH BEALL: What is the total energy in low-energy CRs in the remnants on compared to the total energy of the remnant?

Based on the model described in section团, we estimated the total energy of low-energy CRs for W28, W44, and IC 443 . The total energy of the low-energy protons $(<1000 \mathrm{MeV})$ of the individual SNRs is estimated to be several times $10^{48} \mathrm{erg}$, which is about $1 \%$ of the total CR energy.

\section{Acknowledgments}

The author is most grateful to the organizers for their kind invitation to give this talk at this fantastic workshop. The author wishes to acknowledge my collaborators: Dr. Masayoshi Nobukawa, Dr. Shigeo Yamauchi, Dr. Yutaka Fujita, Dr. Katsuji Koyama, and also two bright graduate students, Arisa Hirayama and Ken Makino. This research is supported by Research Fellowships of the Japan Society for the Promotion of Science (JSPS) for Young Scientists.

\section{References}

[1] F. Acero et al., 2016, The First Fermi LAT Supernova Remnant Catalog, ApJS 224 (1) 8

[2] H.E.S.S. Collaboration. 2018, Population study of Galactic supernova remnants at very high $\gamma$-ray energies with H.E.S.S., A\&A 612 A3

[3] N. Indriolo et al., 2015, Herschel Survey of Galactic $\mathrm{OH}^{+}, \mathrm{H}_{2} \mathrm{O}^{+}$, and $\mathrm{H}_{3} \mathrm{O}^{+}$: Probing the Molecular Hydrogen Fraction and Cosmic-Ray Ionization Rate, ApJ 800 (1) 40

[4] E. C. Stone et al., 2013, Voyager 1 Observes Low-Energy Galactic Cosmic Rays in a Region Depleted of Heliospheric Ions, Science 341 (6142) 150

[5] K. Lodders, 2003, Solar System Abundances and Condensation Temperatures of the Elements, ApJ 591 (2) 1120

[6] T. Sato et al., 2014, Discovery of recombining plasma in the supernova remnant 3C 391, PASJ 66 (6) 124

[7] T. Sato et al., 2016, Suzaku spectra of a Type-II supernova remnant, Kes 79, PASJ 68 (SP1) S8

[8] K. K. Nobukawa et al., 2018, Evidence for a Neutral Iron Line Generated by MeV Protons from Supernova Remnants Interacting with Molecular Clouds, ApJ 854 (2) 87 
[9] B. Jiang et al., 2010, Cavity of Molecular Gas Associated with Supernova Remnant 3C 397, ApJ 712 (2) 1147

[10] V. Dogiel et al., 2011, K-Shell Emission of Neutral Iron Line from Sagittarius B2 Excited by Subrelativistic Protons, PASJ 63 (3) 535

[11] S. Saji et al., 2018, Discovery of $6.4 \mathrm{keV}$ line and soft X-ray emissions from G323.7-1.0 with Suzaku, PASJ 70 (2) 23

[12] S. Saji, 2018, Suzaku Study of Non-Thermal Iron Line Emission at 6.4 keV from Supernova Remnants, Ph.D. dissertation, Nagoya University

[13] A. Bamba et al., 2018, The Transition from Young to Middle-aged Supernova Remnants: Thermal and Nonthermal Aspects of SNR N132D, ApJ 854 (1) 71

[14] K. K. Nobukawa et al., 2019b, Neutral iron line in the supernova remnant IC 443 and implications for MeV cosmic rays, PASJ 71 (6) 115

[15] H. Yamaguchi et al., 2009, Discovery of Strong Radiative Recombination Continua from the Supernova Remnant IC 443 with Suzaku, ApJL 705 (1) L6

[16] T. Ohnishi et al., 2014, Discovery of Enhanced Radiative Recombination Continua of He-like Iron and Calcium from IC 443 and its Implications, ApJ 784 (1) 74

[17] K. K. Nobukawa et al., 2019a, Measurement of Low-Energy Cosmic Rays via the Neutral Iron Line, in proceedings of 26th Extended European Cosmic Ray Symposium, Journal of Physics: Conference Series 1181012040

[18] K. Makino et al., 2019, Interaction between molecular clouds and MeV-TeV cosmic-ray protons escaped from supernova remnants, PASJ 71 (4) 78

[19] Albert et al., 2007, Discovery of Very High Energy Gamma Radiation from IC 443 with the MAGIC Telescope ApJL 664 (2) L87

[20] Acciari et al., 2009, Observation of Extended Very High Energy Emission from the Supernova Remnant IC 443 with VERITAS ApJL 698 (2) L133

[21] Abdo et al., 2010, Observation of Supernova Remnant IC 443 with the Fermi Large Area Telescope ApJ 712 (1) 459

[22] Y. Ohira et al., 2011, Gamma-rays from molecular clouds illuminated by cosmic rays escaping from interacting supernova remnants MNRAS 410 (3) 1577 